\title{
Free Switching System of 3D Animated Gradient Scene Based on Virtual Reality
}

\author{
Zheng Jinming ${ }^{\text {a, }}$, Huang Chen, Jiang Zhifeng \\ Wenzhou Academy of Agricultural Sciences (Wenzhou Vocational College of Science and Technology, \\ Institute of Information Technology), Wenzhou, 325000, Zhejiang, China \\ a email: kimizh14@163.com \\ *corresponding author
}

Keywords: Virtual Reality, 3D Animation, Gradient Scene, Free Switching System

\begin{abstract}
With the continuous improvement of modern social and economic level and the rapid development of science and technology, more and more advanced technologies have emerged and applied in various fields, gradually changing people's way of life, work and entertainment. Virtual reality technology is a technology with strong application, which can meet the needs of various fields. In the design and production of 3D animation, technicians apply it to the free switching of animation gradient scene, and construct the free switching system to strengthen the 3D animation effect and bring a better experience to customers. This paper briefly expounds the technical overview and basic characteristics of virtual reality, analyzes the virtual reality technology in the 3D animation gradient scene, and probes into the construction strategy of the 3D animation gradient scene free switching system.
\end{abstract}

\section{Overview and Basic Characteristics of Virtual Reality Technology}

Since the last century, the rapid development of computer network technology in China, people's daily entertainment and leisure mode has undergone earth-shaking changes, the traditional animation form has gradually been replaced by 3D animation, more novel animation expression into people's vision. In recent years, the level of 3D animation design and production technology in China has been continuously improved, and technicians have made full use of the interactive, immersive, interactive and simulation of virtual reality technology to construct real animation scenes, and urge people to be able to produce the feeling of "immersive ". Based on this, technicians combine the characteristics of virtual reality technology, according to the $3 \mathrm{D}$ animation gradual change scene design requirements, build a free switching system, so as to strengthen the scene switching performance, promote the scene change more natural, provide people with a better animation viewing feeling.

\subsection{Overview of virtual reality technology}

Virtual reality technology is an important component of modern social simulation technology. The technology personnel combine computer graphics death, man-machine interface technology, sensing technology, simulation technology, network technology and multimedia technology to form a rich and challenging cross-type technology, which can be widely used in various fields, such as: scene design, animation production and so on. Virtual reality technology can also be called "VR technology "(Figure 1), which includes: perception, natural skills, simulation environment, sensing function and so on. The simulation environment is mainly generated by computer, and it is a more realistic three-dimensional image [1]. Perception and natural skills are the ability to have all human perception, such as: smell, touch, hearing and so on, but also to achieve the human eye, gestures, movements and so on. 


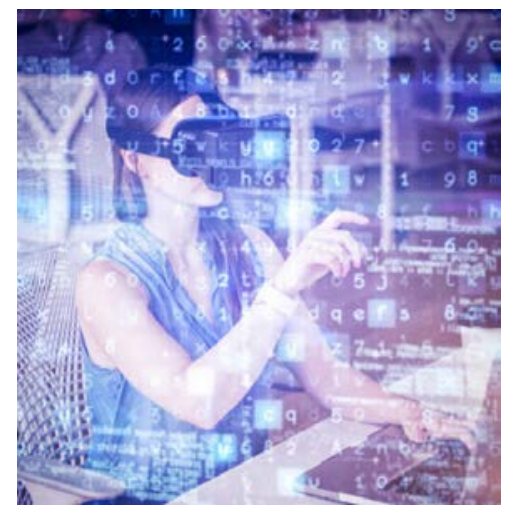

Figure 1 Virtual reality system use pictures

\subsection{Basic characteristics of virtual reality technology}

In daily life, virtual reality technology is also called "spiritual realm technology ", which has three basic characteristics:

With interactive characteristics, people can use this technology to strengthen the animation viewing experience, break through the limitations of traditional animation, communicate with the characters in the animation, touch the objects in the animation scene;

In the process of applying virtual reality technology, the technicians combine computer application technology, image technology and computer flow technology to construct the real animation scene environment, and make people feel that they are "in the animation" and produce better visual experience and auditory feeling [2].

With the characteristics of conception, technicians use virtual reality technology to construct animation scene, customers can experience the real world in the animation scene, but also can combine their own active imagination to interact reasonably with the surrounding environment.

\section{Virtual Reality Technology in 3D Animation Gradient Scene}

\subsection{Follow the Basic Design Principles}

In the design and production of 3D animation gradient scene, technicians should strictly follow the basic principles of animation design by using virtual reality technology. First, technicians should follow the principle of interesting design, ensure the entertainment of animation, and relieve people's work pressure through animation. If the animation scene design is not interesting, it will affect the customer's viewing feeling, can not attract the attention of the customer, and reduce the virtual reality design utility of the 3D animation gradient scene.[3]. Second, to follow the cultural principle, 3D animation is the development and innovation of traditional animation in our country in the new period. Whether it is the whole design or the gradual scene design, it should follow the design and production idea of the traditional animation in our country, that is ", having a certain humanism ", which can spread certain cultural ideas through animation and scene, such as the existence of ancient Chinese architecture in the gradual scene, which can convey Chinese traditional culture. Third, we should follow the beneficial principle, if the gradual change scene in 3D animation is too violent and bloody, it is not suitable for children to watch, but also for adults. This is mainly due to the strong immersion of $3 \mathrm{D}$ animation, which will bring real viewing feeling to customers (Fig .2). Fourth, innovative principle, in the process of 3D animation gradual change scene design, technicians should constantly improve the scene content, to innovate the animation picture, improve the overall level of animation, and further strengthen the customer's viewing experience. 


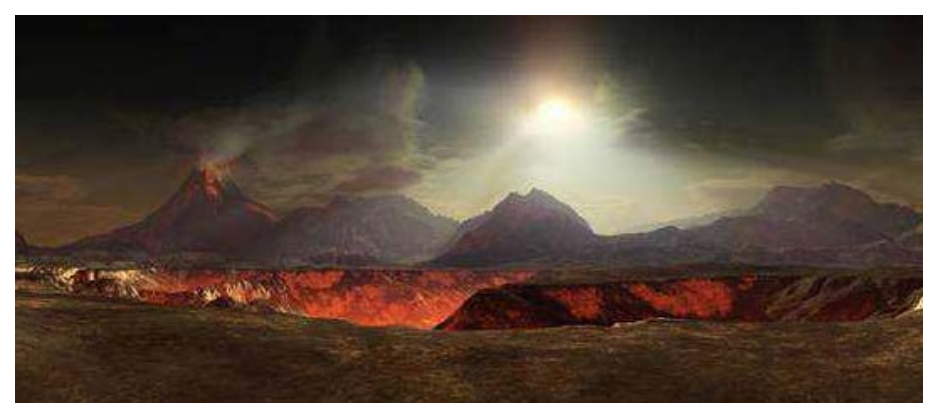

Figure 2 3D animated gradient scene works of virtual reality technology

\subsection{Designing a sci-fi animation scene}

In the design and production of 3D animation gradient scene, technicians should pay attention to the scene gradient design in science fiction animation. In the process of design, technicians should reasonably use virtual reality technology to analyze from the perspective of customer viewing, so as to urge customers to be able to better immerse themselves in 3D animation through sci-fi scenes, and produce more real sci-fi animation viewing feelings. Among the many types of animation, it is more difficult for science fiction animation to design gradually and freely, so it is necessary for technicians to constantly coordinate the relationship between virtual reality technology and actual animation design, but also to control the audience's psychological needs and optimize the details in the gradual scene, so as to make the scene more realistic, free switching more smooth and natural, so that customers can have a better experience, meet the needs of 3D science fiction animation, and further improve the market competitiveness of 3D animation [4]. In the process of science fiction animation gradually becoming fine design, technicians should determine the scene style in advance, urge customers to better understand the animation theme, through science fiction refined, stimulate the enthusiasm of customers to watch, actively explore the mysterious unknown world.

\subsection{Designing Adventure Animation Scenes}

In the design and production of 3D animation gradient scene, technicians should also pay attention to the 3D animation gradient scene design of adventure action class. In the traditional adventure animation scene, the customer can complete his imagination and thinking through the animation picture, the viewing experience is relatively single, and only stay in the plane visual stimulation level, the user can not experience the animation hero in the adventure process of excitement and expectation. After integrating into the virtual reality technology, the $3 \mathrm{~d}$ scene gradually becomes the main formation of animation scene design, which can not only provide the real adventure experience for the customer, but also promote the customer to feel the exciting adventure process like the protagonist of the animation, but also produce the visual impact and construct the all-round animation adventure experience. Based on this, in the design of the gradual scene of 3D adventure animation, technicians should combine different customer groups to carry out targeted design to meet the needs of customers' adventure experience, and also control the authenticity of the scene, so that customers can better integrate into the scene in the process of viewing, and naturally see different scenery in the process of scene switching, so as to create a good, interesting and experiential adventure scene animation for customers.[5].

\section{The Construction Strategy of Virtual Reality's 3D Animated Gradient Scene Free Switching System}

\subsection{Establishing a 3D animation gradient scene model}

To construct the 3D animation gradient scene free switching system of virtual reality, we must first combine the animation design and production requirements to establish the corresponding scene model (figure 3). Technicians should combine virtual reality technology and 3D animation production technology to complete the modeling of scene and scene, and integrate human-computer interaction function into it, so as to ensure that the scene is more authentic. In the process of 
constructing the model in practice, technicians should collect a large number of relevant elements of the scene, such as buildings, natural scenery and so on, and organize and summarize the data to construct the scene as the basic data, and keep the deformation process of the object in the scene as the main data of free switching, so as to improve the flexibility and naturalness of the free switching of the animation gradual scene.

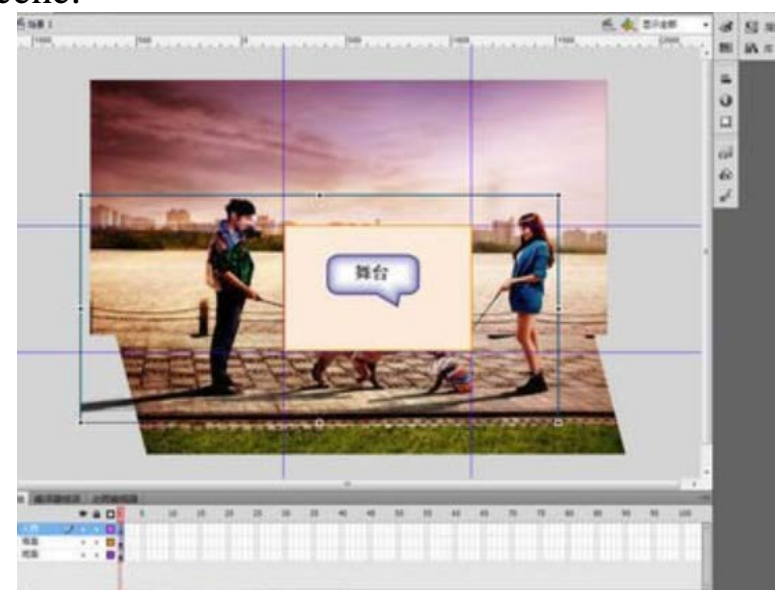

Figure 33 Construction of gradient scene model for animation

\subsection{Capture 3D animation details}

To construct the 3D animation gradient scene free switching system, it is necessary to capture the background and the object change in the 3D animation gradient scene in detail, use the object change movement information, combine the virtual reality technology, according to the data after the computer technology processing, carry on the adjustment and disposal of the object shape, so as to meet the free switching demand of the gradual change scene of the animation, ensure that the lens can be placed in any place in the process of animation production, and promote people to have a shocking sense of substitution when watching the animation in the later stage.

\subsection{Adjust the gradient scene in 3D animation}

In order to construct the 3D animation gradient scene free switching system, it is necessary to adjust properly and play the role of virtual reality technology in 3D animation. In this process, in order to better complete the free switching of the gradient scene and construct the free switching system, the technicians can use the superposition method and the insertion method to modify the details of the picture and the animation gradient scene, and ensure that the data can meet the switching needs of the animation scene through repeated adjustment.[6].

\section{Conclusion}

To sum up, combined with the basic characteristics and application advantages of virtual reality technology, technicians should follow three basic principles in the process of constructing the free switching system of 3D animation gradient scene, and also focus on adjusting the scene design of science fiction animation and adventure animation to enrich the texture of the picture. In addition, technicians should ensure the rationality and scientific nature of the 3D animation gradient scene switching, construct the gradient scene model, capture the details of the animation picture, adjust the change of the gradient scene in the animation, so as to enhance the overall level of the 3D animation and provide customers with a better viewing experience.

\section{Acknowledgements}

"Research on Agricultural Scene Visualization Based on Virtual Reality Technology and Visual Communication Technology" from Wencheng County Science and Technology Bureau of Zhejiang Province in 2019 (2019NKYO8);

"Analysis, prediction, and automatic judgment on public opinions from WeChat public account 
based on artificial intelligence technology" from Wenzhou Science and Technology Bureau of Zhejiang Province in 2019 (S20190014).

\section{References}

[1] Zhang Honglei. Virtual reality (VR) under 5 G. Computer Products and Circulation, no. 01, pp. 120, 2020.

[2] Sun Hongxing. Virtual reality key technology application and research. Think Tank Age, no. 01, pp. 244-245, 2020.

[3] Li Jing. Automatic planning of scene material in mobile phone 3D animation. Computer Systems Applications, vol. 28, no. 06, pp. 53-61, 2019.

[4] Fu Jianming, Bao Yan. 3D animation scene graphic design based on virtual reality technology. Modern Electronic Technology, vol. 40, no. 21, pp. 59-61+65, 2017.

[5] Yang Junjie. Application of $3 \mathrm{D}$ animation and virtual reality technology. Information and Computers (Theoretical Edition), no. 02, pp. 190-191, 2017.

[6] Chen Huazhong. New Feel Flash MX2004 Serial III: Animation Production. Computer Knowledge and Technology, no. 25, pp. 59-62, 2004. 